\title{
Modeling in solving application-oriented tasks as the basis for the formation of professional competence of future computer science and information technology teacher
}

\author{
Z.A. Kononova ${ }^{1, *}$ and S.O. Altukhova ${ }^{1}$ \\ ${ }^{1}$ Institute of Natural, Mathematical and Engineering Sciences Lipetsk State Pedagogical University \\ named after P.P. Semenov-Tyan-Shanskiy, Lipetsk, Russia
}

\begin{abstract}
The article is devoted to the study of matters in solving applied tasks in computer science by using methods of mathematical and computer modeling. These methods allow to create an innovative approach to solving research tasks that are the basis for the development of key competencies of the future teacher of computer science. The article provides an example of building a model of "predator"-"prey", which includes: a general description of the model, stating the problem, compilation of a mathematical model for dealing with the task, transferring a mathematical model into a computer one. Modeling in the field of dealing with applied tasks is actively used while teaching students at Lipetsk State Pedagogical University named after P.P. Semenov-Tyan-Shanskiy.
\end{abstract}

\section{Introduction}

Contemporary trends in any professional field are focused not on training qualified personnel, but on the personnel who are themselves able to prepare and implement various methods, technics, technological processes and so on in their professional activities. The training of the future specialists who can inspire, show, teach must begin from the school bench. And in this case such a subject as computer science can claim a leading role.

In high school, computer science is viewed as a subject aimed at studying general patterns. The use of information systems in teaching allows us to develop and expand the possibilities of a systemic vision of the world, establish cross-curricular connections of computer science with other disciplines, form research activities in dealing with applied tasks, utilizing the benefits of computer science and ICT in general. Prime examples of that statement are such segments of computer science as "Information models" and "Information systems". But in order to achieve the result, it is necessary while training a future computer science teacher to pay special attention to programming courses devoted to the practical problems in the modeling of various processes and phenomena [1]. This approach has been implemented for several years at the Lipetsk State Pedagogical University named after

\footnotetext{
* Corresponding author: kononovazoy@gmail.com
} 
P.P. Semenov-Tyan-Shanskiy while teaching students at the faculty of "Pedagogical Education" specializing in "Computer Science".

The most common scope of application for modeling is science. Mathematical and computer models are used in pharmacology, ecology, biology, chemistry and so on. It is also promising to use modeling in manufacturing and economics. Modeling of social processes, individual behavior, intergroup relations is slightly more challenging. Based on the above, the models can be classified according to the area of use, the time factor, the area of knowledge, the way of presentation. It should be noted that modeling enables integrating science and practice in various fields [2-4]. This is the key for the effective development of the educational process. Gaining of modeling skills in high school or university can become the basis for the integration of theory and practice, both within the framework of one subject or several disciplines [5]. Let us consider the problem of building a model of biological phenomena. At present, the reproduction of various biological processes in artificial systems is gaining wide popularity. The main task of such modeling is experimental verification of hypotheses about the structure and functions of biological systems.

\section{The predator-prey model}

\subsection{General description of the model}

In the simplest case, "prey" is the only food for the predator. In this scenario, the disappearance of the prey leads to the disappearance of the predators, in contrast to the model of interspecific competition, when the disappearance of one population leads to the growth of another. The number of the prey population is denoted by $\mathrm{N}$, the number of the predator population is denoted by $\mathrm{C}$. Taking into account the influence of prey and predators on each other, we obtain the following model $[6,7]$ as in system of equations $(1)$ :

in which:

$$
\left\{\begin{array}{l}
\frac{\mathrm{dN}}{\mathrm{dt}}=R \cdot N-a \cdot C \cdot N \\
\frac{\mathrm{dC}}{\mathrm{dt}}=f \cdot a \cdot C \cdot N-q \cdot C
\end{array}\right.
$$

$\frac{\mathrm{dN}}{\mathrm{dt}}$ - the rate of change in the number of victims;

$\mathrm{R}$ - the rate of reproduction of victims;

a - the rate of efficiency of predators' search for prey;

$\frac{\mathrm{dC}}{\mathrm{dt}}$ - the rate of change in the number of predators;

$\mathrm{f}$ - the rate of efficiency of the transition of food into the offspring of predators;

$\mathrm{q}$ - the rate of decline in the population of predators in the absence of prey.

In this model, there is no direct rate of reproduction of predators since it is related to the parameters f, a, $\mathrm{N}(f \cdot a \cdot N)$.

Thus, in the absence of predators $(\mathrm{C}=0)$, the number of prey grows exponentially at a rate $\mathrm{R}$, so we achieve a population growth model without taking into account intraspecific competition. The growth rate of the number of victims $\frac{\mathrm{dN}}{\mathrm{dt}}$ decreases with an increase in the frequency of encounters with prey and predators, that reflects the coefficient a. In the absence of prey, the number of predators decreases exponentially with a rate of q, i.e. composition $(f \cdot a \cdot N)$ should compensate for this decline in numbers or even increase the number of predators.

\subsection{Stating the problem}


- Calculate the dynamics of populations of predators and prey (for instance Arctic foxes and lemmings, pike and crucian carp).

- Demonstrate the dependence of the size of both populations (prey and predators) at time for certain values of the parameters $\mathrm{R}, \mathrm{a}, \mathrm{q}$ and $\mathrm{f}$. The results are illustrated in graphic form.

- Demonstrate the dependence of prey and predators at rate of prey reproduction. The results of the evolution of populations are presented in graphic form and in the form of a dynamic picture.

\subsection{Compilation of a mathematical model for dealing with the task}

When modeling a complex processes the influence of a large number of parameters should be taken into account. Therefore, it is more convenient to study a set of simpler components of the process. Mathematical apparatus is of great significance in modeling. Various mathematical models can be implemented and used to study the static and dynamic properties of any scale in stationary or non-stationary modes of operation to determine various characteristics and parameters depending on the task for the same object of modelling. Using the modeling makes it possible to study both general and particular patterns of macro- and microsystems, their response to internal and external changes [8]. In contemporary studies information theory, mathematical logic, probability theory and others are combined with the methods of mathematical statistics [9].

Let us consider the process of compiling a mathematical model using the example cited the present study [10].

The number (concentration) of predators and prey are denoted by letters $\mathrm{C}$ and $\mathrm{N}$, respectively. Supposing the victims have sufficient amount of food, in the scenario were there are no predators, the increase in herbivores would be proportional to the number of the currently available population as in equation (2):

$$
d t=R \cdot N
$$

In the presence of predators, the number of herbivores consumed by them is proportional to the number of interactions between both species of animals. Assuming this, a differential equation can be created for the changing number of herbivores as in equation (3):

$$
d t=R \cdot N-a \cdot N \cdot C
$$

If mortality among predators is natural, then it is proportional to the number of predators $(-q \cdot C)$. The increase in the population of predators is proportional to their number and the amount of food (i.e. the number of herbivores): $f \cdot a \cdot N \cdot C$. Hence, the differential equation describing the dynamics of the predator population is the following as in equation (4):

$$
d t=-q \cdot C+f \cdot a \cdot N \cdot C
$$

If we use the same model, but to describe the dynamics of populations of carnivorous and herbivorous fish, assuming that the number of both fish species additionally decreases as a result of fishing, the following system of differential equations will be obtained as in equations (5-6):

$$
\begin{aligned}
& \frac{d N}{d t}=R \cdot N-a \cdot N \cdot C-f \cdot N \\
& \frac{d C}{d t}=-q \cdot C+f \cdot a \cdot N \cdot C-f \cdot C
\end{aligned}
$$


Catches (quotas), which are less than the growth parameter of herbivorous fish N, increase the average proportion of herbivorous fish and decrease the proportion of predatory fish. Thus, if fish were not caught at all, then the relative number of herbivorous fish, most of which are commercial fish, would decrease, and the number of predatory fish would increase (Volterra's principle).

Based on the above statements, we will describe the model of the "predator-prey" system in details by having successively performed algebraic actions to prepare an answer to the system of differential equations as in systems of equations (7-10):

$$
\begin{gathered}
\left\{\begin{array}{c}
d N=(R \cdot N-a \cdot C \cdot N) \cdot d t \\
d C=(f \cdot a \cdot C \cdot N-q \cdot C) \cdot d t
\end{array}\right. \\
\left\{\begin{array}{l}
N=\int d N \\
C=\int d C
\end{array}\right. \\
\left\{\begin{array}{c}
N=N+(R \cdot N-a \cdot C \cdot N) \cdot d t \\
C=C+(f \cdot a \cdot C \cdot N-q \cdot C) \cdot d t \\
t=t+d t
\end{array}\right. \\
\left\{\begin{array}{c}
N[i]=N[i-1]+(R \cdot N[i-1]-a \cdot C[i-1] \cdot N[i-1]) \cdot d t \\
C[i]=C[i-1]+(f \cdot a \cdot C[i-1] \cdot N[i-1]-q \cdot C[i-1]) \cdot d t \\
t[i]=t[i-1]+d t
\end{array}\right.
\end{gathered}
$$

\subsection{Translation of a mathematical model into a computer model}

The improvement of computer technologies gives additional opportunities for solving practical problems, including modeling various objects, phenomena or processes by increasing the accuracy and speed of computation.

Building a model is a rather laborious process, which includes not only understanding the object itself, phenomenon or process and the laws of their change, but also a complex calculation part. Often these challenges are associated with the definition of derivatives and calculations of integrals of complex functions [11]. At the very beginning of the development of electronic computing technology, these calculations were carried out based on approximate and numerical methods which are based on the division principle of function parameter being researched into a finite number of steps. The present situation in science and technology, in particular, computer science, makes it possible to solve applied problems without using approximate computational methods where the segment of the function parameter under research was divided into a finite number of steps. The present situation in science and technology in particular computer science enables to solve applied problems without using approximate computational methods $[12,13]$.

To prove the above mentioned statement, let us consider an arbitrary function $y=f(x)$ on the interval $[X 1, X n]$. Integral of a function $y=f(x)$ on this interval there is an area of a curvilinear trapezoid bounded along the OY axis by the function $y=f(x)$ itself, and along the OX axis - by the coordinates X1 и Xn (Figure 1).

The area of a curvilinear trapezoid can be calculated as the sum of a number of elementary trapezoids with a base $\Delta \mathrm{x}$. Then, denoting the required integral as $\mathrm{S}$, we can conclude that $S=\sum_{i=1}^{n} S_{i}$ (Figure 2). 


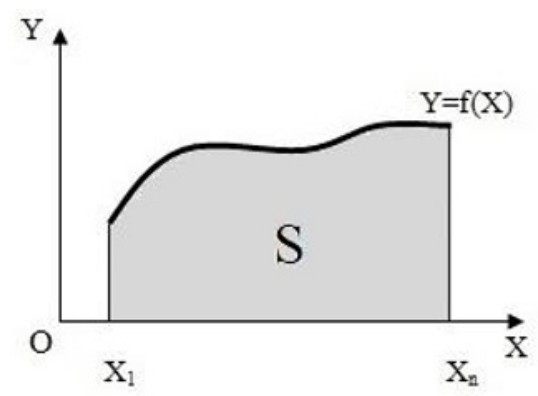

Fig. 1. Arbitrary function.

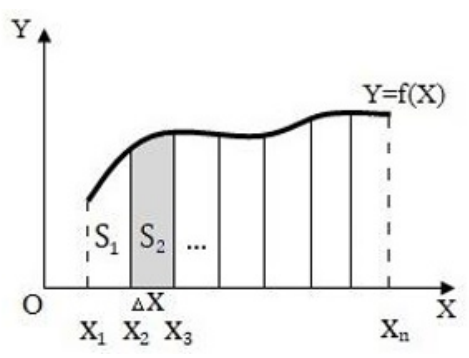

Fig. 2. Graphic interpretation of the integral.

The calculation of the sum by programming methods is carried out using any loop operator: a loop with a parameter or loops with a condition. Table 1 shows examples of using conditional loop operators to solve the problem. The presented options for calculating the area of a curvilinear trapezoid are preferable, because in both examples, not the number of calculation iterations is indicated, but in the first case it is the condition for achieving the limits of the variable (parameter), and in the second case performing calculations with a specified error.

Table 1. Examples of calculation of the amount.

\begin{tabular}{|c|c|}
\hline $\begin{array}{l}\text { Example 1. Reaching variable } \\
\text { limits (Parameter) }\end{array}$ & $\begin{array}{l}\text { Example 2. Perform calculations with } \\
\text { specified error }\end{array}$ \\
\hline $\begin{array}{l}\text { readln }(x[1]) ; \\
\text { readln }(x n) ; \\
\text { readln }(\mathrm{dx}) ; \\
\mathrm{s}[0]:=0 ; \\
\mathrm{i}:=1 ; \\
\text { While } \mathrm{x}[\mathrm{i}]<\mathrm{xn} \text { do } \\
\text { begin } \\
\quad \mathrm{y}[\mathrm{i}]:=\mathrm{sqr}(\mathrm{x}[\mathrm{i}]) \\
\mathrm{s}[\mathrm{i}]:=\mathrm{s}[\mathrm{i}-1]+\mathrm{y}[\mathrm{i}]^{*} \mathrm{dx} \\
\mathrm{i}:=\mathrm{i}+1 ; \\
\mathrm{x}[\mathrm{i}]:=\mathrm{x}[\mathrm{i}-1]+\mathrm{dx} ; \\
\text { end; }\end{array}$ & $\begin{array}{l}\text { readln }(\mathrm{x}[0]) ; \\
\text { readln }(\mathrm{eps}) ; \\
\text { readln }(\mathrm{xn}) ; \\
\text { readln }(\mathrm{dx}) ; \\
\mathrm{i}:=0 ; \\
\mathrm{y}[\mathrm{i}]:=\exp (\mathrm{x}[\mathrm{i}]) / \operatorname{sqrt}(\mathrm{x}[\mathrm{i}]) ; \\
\text { Repeat } \\
\quad \mathrm{i}=\mathrm{i}+1 ; \\
\quad \mathrm{x}[\mathrm{i}]:=\mathrm{x}[\mathrm{i}-1]+\mathrm{dx} ; \\
\mathrm{y}[\mathrm{i}]:=\exp (\mathrm{x}[\mathrm{i}]) / \mathrm{sqrt}(\mathrm{x}[\mathrm{i}]) ; \\
\mathrm{Until} \text { abs }(\mathrm{y}[\mathrm{i}]-\mathrm{y}[\mathrm{i}-1])<\mathrm{eps} ;\end{array}$ \\
\hline
\end{tabular}

If we reduce the base of the elementary trapezoid $\Delta \mathrm{x}$ to values close to zero, then we can gradually move to the differential dx (Figure 3 ).

When $d x \rightarrow 0$ the function $y=f(x)$ at each point of the curve tends to a constant value. Consequently, the function can be divided into an infinite number of constants. From the discrete value of the sum $S=\sum_{i=1}^{n} S_{i}$ we can proceed to the continuous sum value, in the present scenario, the elementary areas $\mathrm{dS}=f\left(x_{i}\right) \cdot \mathrm{dx}[14]$.

The implementation of the considered mathematical model in a computer is presented in the Embarcadero Delphi programming environment. This environment allows you to create a convenient user interface, and display the results of calculations both in tabular form and in graphical form. For the students of pedagogical education specializing in "Computer Science", the choice of this programming environment is not challenging, since this environment and programming skills are studied as disciplines "Languages and Methods of Programming", "Object-Oriented Programming", "Programming in High-Level Languages" (depending on the name of the subject in the curriculum). In addition to displaying the simulation results in a graphical form (Figure 4), the so-called "dynamic picture" is shown, 
that is, in a ranged time mode, a dynamic change in the number of prey and predators is shown in a certain ranged population habitat (Figure 5).

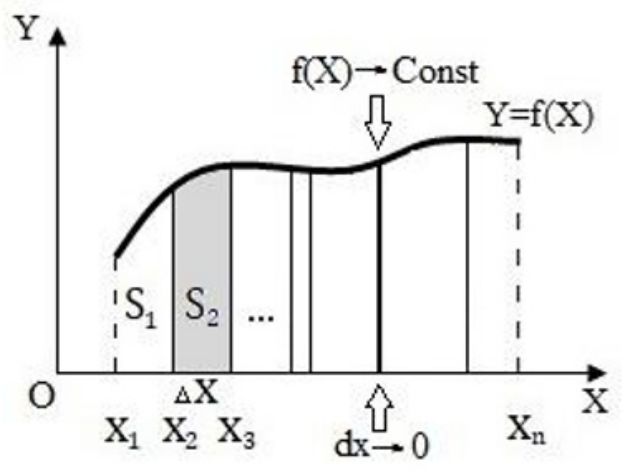

Fig. 3. An example of a gradual decrease in the discrete interval $\Delta x$ to infinitesimal $d x$.

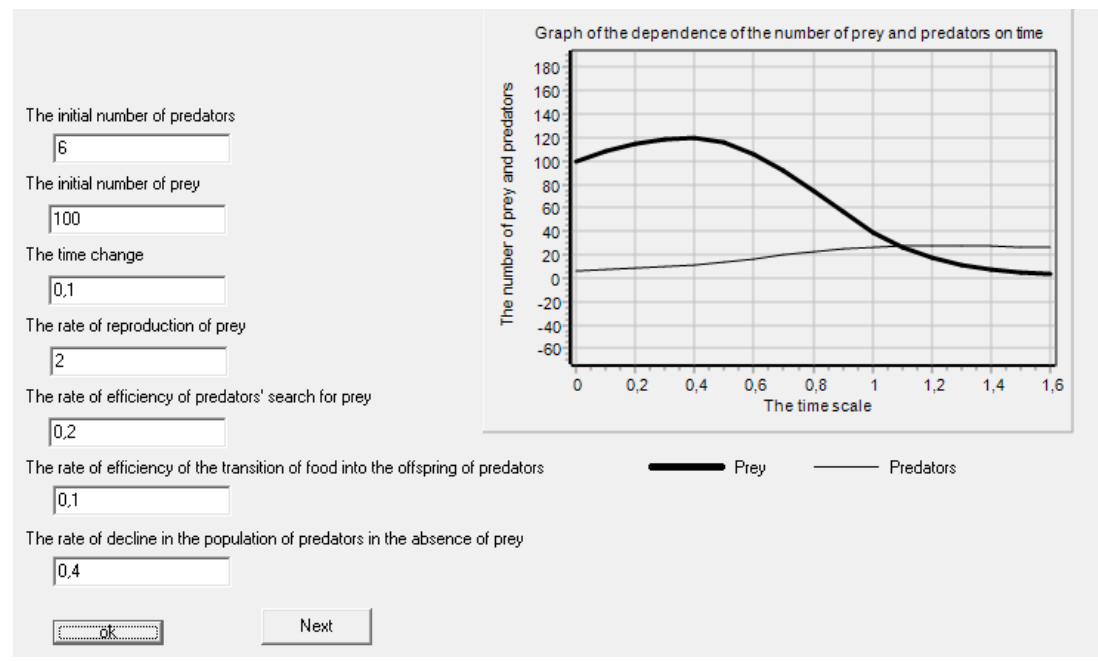

Fig. 4. Graph of the dependence of the number of prey and predators on time.

A thin line will correspond to predators whereas a thick line will correspond to victims. It also shows the influence of the value of the rate of natural increase in prey on the development of the population at a given time [15].

\section{Conclusion}

The students of Pedagogical Education with a specialization of "Computer Science" at the Lipetsk State Pedagogical University named after P.P. Semenov-Tyan-Shanskiy develop skills of building similar models for solving problems in various fields, including chemistry, biology, physics.

Thus, the skills and abilities of building models will save the students significant resources, directing them to the study of a specific problem, rather than "scattering" to find the optimal solution. In addition, modeling has become the basis for the integration of various scientific disciplines, along with theory and practice. Therefore, it is crucial to develop such capabilities. Indeed, students who have mastered modelling become "universal" specialists 
who can successfully solve a wide range of socio-economic, industrial and scientific problems.

Modeling enables the integration of science and practice in various fields. This is especially vital for the effective progress in the educational process. The acquisition of the modeling skills in high school (especially important for specialized classes), and then at the university, can become the basis for the integration of theory and practice (both within the framework of one or several academic subjects) in addition to the formation of practical tools in the preparation and in implementing research.

\section{References}

1. Markovich O S, Sergeev A N 2019 RUDN Journal of Informatization in Education 16(4) 351

2. Pavlovsky Y N, Belotelov N V. Brodsky Y.I. 2008 Simulation modeling (Moscow: ACADEMA) p 234

3. Kolesov Y B, Senichenkov Y B 2006 System modeling. Dynamic and hybrid systems (SPb: BHV-Peterburg) p 226

4. Shannon E R 1978 Systems simulation: The Art and Science (Moscow: MIR) p 418

5. Abdurazakov M M, Dordjpalam O 2017 Baltic humanitarian magazine 4(21) 223

6. Glukhov V V, Lisochkina T V, Nekrasova T P 1997 Economic bases of ecology (SPb: Special'naya literatura) p 304

7. Mogilev A V, Pak N I, Henner E K 2003 Informatics (Moscow: ACADEMA) p 816

8. Sokolowski J A, Banks C M 2009 Principles of Modelling and Simulation (NJ: John Wiley and Sons) p 280

9. Zavarykin V M, Zhytomyr V G, Lapchik M P 1990 Numerical methods (Moscow: Prosveshchenie) p 176

10. Samarsky A A, Mikhailov A P 2005 Mathematical modeling: Ideas. Methods. Examples (Moscow: Nauka) p 320

11. Demidovich B P, Maron I A 1966 Fundamentals of Computational Mathematics (Moscow: Nauka) p 664

12. Semakin I G, Rusakova O L, Tarunin E L, Shkaraputa A P 2017 Programming, numerical methods and mathematical modeling (Moscow: KNORUS) p 304

13. Fishwick A P 1995 Simulation Model Design and Execution: Building Digital Worlds (NJ: Prentice Hall) p 448

14. Kononova $\mathrm{Z}$ A Calculation of integrals on a computer 2008 Issues of lifelong education: materials of the International scientific-practical conference, Lipetsk, LGPU

15. Kononova Z A, Altukhova S O, Vorobiev G A 2018 Computer simulation. Ecology: workshop (Lipetsk: Lipetsk State Pedagogical University named after P.P. SemenovTyan-Shanskogo) p 101 